\title{
A NEW SPECIES OF THE GENUS Nyereria Mason (Hymenoptera: Braconidae: Microgastrinae) FROM VIETNAM
}

\author{
Khuat Dang Long ${ }^{1 *}$, Cornelis van Achterberg ${ }^{2}$ \\ ${ }^{1}$ Institute of Ecology and Biological Resources (IEBR), VAST, Ha Noi, Vietnam, \\ *khuatdanglong@iebr.ac.vn \\ ${ }^{2}$ Department of Terrestrial Zoology (RMNH), Naturalis Biodiversity Center, Leiden, Netherlands
}

\begin{abstract}
Nyereria Mason, 1981 is a medium-sized genus of the subfamily Microgastrinae (Braconidae) with two species already known from Vietnam, viz. Nyereria albicentrus Long \& van Achterberg, 2008 and $N$. yenthuyensis Long \& van Achterberg, 2008. In this paper, a new species of this genus is described and illustrated: Nyereria bicolorata Long \& van Achterberg, sp. n.. The checklist and distribution of the twenty three named species are given, of those eighteen species are described from the Afrotropical region and four species from the Oriental region. One species is known from the East Palaearctic region. A key to the species of the genus Nyereria from Vietnam is provided.
\end{abstract}

Keywords: Hymenoptera, Braconidae, Microgastrinae, Nyereria, new species, Vietnam.

\section{INTRODUCTION}

The genus, Nyereria Mason, 1981, is common in the Afrotropical region (more common and diverse than Mason thought when he described the new genus in 1981). In the previous paper, two species of this genus from Vietnam's fauna were described in Protapanteles Ashmead because Nyereria Mason was treated as subgenus of Protapanteles (Long \& van Achterberg, 2008). In this paper, Taxapad (Yu et al., 2012) [16] is followed, where Nyereria is treated as a valid genus.

Particularly, the incorrect locality of the holotype of Nyereria albicentrus Long \& van Achterberg, 2008 indicated in the original description was replaced as written in the type label.

Up to date, the genus Nyereria comprises twenty three species, of which eighteen species occur in the Afrotropical region, four species occur in the Oriental region and one in the East Palaearctic region.

\section{MATERIALS AND METHODS}

Specimens studied are deposited in the Collection of the Institute of Ecology \& Biological Resources (IEBR) and Vietnam National Museum of Nature (VNMN) at $\mathrm{Ha}$
Noi, assembled by the first author during numerous expeditions in Vietnam.

Terminology used in this paper follows van Achterberg (1988) [1], sculpture terms are based on Harris (1979) [5], for a key to the genera of the subfamily Microgastrinae see Nixon (1965) [12], for diagnosis of the genus Nyereria see Mason (1981) [11], for additional references and data to the genus Nyereria see Yu et al. (2012) [16].

All the photographs were made by the first author with a Canon G15 digital camera attached to an Olympus ${ }^{\circledR}$ SZ61 binocular microscope; the measurements of wasps were made under an Olympus ${ }^{\circledR} S Z 40$ binocular microscope. Type specimens (holotypes and paratypes) are kept in the Vietnam National Museum of Nature (VNMN), Ha Noi, Vietnam. VAST stands for Vietnam Academy of Science and Technology. Apan.+number and Mic.+number: code number indexing for specimens of the Microgastrinae in the collection. Abbreviations used in this paper are as follows: $\mathrm{AMNH}=$ American Museum of Natural History (USA); IEBR=Institute of Ecology \& Biological Resources (Ha Noi, Vietnam); $\mathrm{RMNH}=$ Netherlands Centre for Biodiversity Naturalis (Leiden, The Netherlands). VNMN=Vietnam National Museum of Nature (Ha Noi, Vietnam); $\mathrm{NC}=$ 
North Central; NE=Northeast; NW=Northwest; $\mathrm{MT}=$ Malaise $\operatorname{trap}(\mathrm{s}) ; \quad \mathrm{POL}=$ postocellar line; $\mathrm{OOL}=$ ocular-ocellar line; $\mathrm{OD}=$ diameter of posterior ocellus; NW: Northwest.

\section{RESULTS AND DISCUSSION}

\section{Systematics}

Nyereria Mason, 1981 (Figs 1-15)

Nyereria Mason, 1981: 108. Type-species (by original designation): Apanteles mlanje Wilkinson, 1929.

\section{Diagnosis}

Nyereria possesses second metasomal tergite divided into three sections by a pair convergent groves, the delimited raised central area distinctly narrowed posteriorly (figs 1, 10, 13); first metasomal tergite parallel-sided (figs 1, 10, 14); hypopygium short with a median fold but without creases; ovipositor sheath short with setae near apex; ovipositor short, thick basally and abruptly tapered near middle length (fig. 7). The propodeum of Vietnamese species is coarsely rugose with a median carina (fig. 3), rugose or smooth and the median area of the second tergite is basally wider than the apex of the first tergite, which make them more similar to Diolcogaster Ashmead, 1900, and to Protapanteles Ashmead, 1898.

Checklist and distribution of Nyereria species

Nyereria achaeus (de Saeger, 1944), from Rwanda;

Nyereria albicentrus Long \& van Achterberg, 2008, from Vietnam;

Nyereria ankaratrensis (Granger, 1949), from Madagascar;

Nyereria areatus (Granger, 1949), from Madagascar;

Nyereria bicolorata Long and van Achterberg, sp. n., from Vietnam;
Nyereria bifissus (de Saeger, 1944), from Democratic Republic of Congo;

Nyereria circinus (de Saeger, 1944), from Democratic Republic of Congo;

Nyereria epaphus (de Saeger, 1944), from Democratic Republic of Congo, Rwanda;

Nyereria flavotorquatus (Granger, 1949), from Madagascar;

Nyereria forensis Tobias, 1977, from Korea, Russia (Khabarovsk Kray);

Nyereria ganges Rousse \& Gupta, 2013, from Reunion Island;

Nyereria geometrae (Granger, 1949), from Madagascar;

Nyereria hiero (de Saeger, 1944), from Democratic Republic of Congo;

Nyereria mayurus Rousse \& Gupta, 2013, from Reunion Island;

Nyereria menuthias (Wilkinson, 1935), from Madagascar;

Nyereria mlanje Wilkinson, 1929, from Democratic Republic of Congo, Malawi, Senegal, Uganda;

Nyereria neavei (Wilkinson, 1929), Democratic Republic of Congo, Malawi;

Nyereria neleus (de Saeger, 1944), from Democratic Republic of Congo;

Nyereria osiris (de Saeger, 1944), from Cameroon; Democratic Republic of Congo, Rwanda;

Nyereria rageshri Sathe, 1988, from India; Nyereria tereus (de Saeger, 1944), from Rwanda;

Nyereria triptolemus (de Saeger, 1944), from Democratic Republic of Congo, Ivory Coast, Rwanda;

Nyereria yenthuyensis Long \& van Achterberg, 2008, from Vietnam.

\section{Taxonomy}

\section{Key to species of the genus Nyereria Mason, 1981 from Vietnam}

1. Ocelli in high triangle (Fig. 2); temple very narrow, in lateral view, transverse width of eye 3.5 times temple width (Fig. 2); first metasomal tergite slightly narrowed apically (Fig. 1); vein 1CU1 of fore wing distinctly longer vein 2-CU1 (Fig. 5); head and propleuron yellow, 
mesoscutum and scutellum pale yellow (holotype), but infuscated in paratype. Nyereria bicolorata sp. $\mathbf{n}$.

- Ocelli in low triangle (Figs 25, 33 in Long \& van Achterberg, 2008)[9]; temple wide, in lateral view, transverse width of eye 1.6-1.7 times temple width (Figs 8, 12); first metasomal tergite parallel-sided (Figs 10, 14); vein 1-CU1 of fore wing slight shorter or subequal to vein 2-CU1 (Figs 11, 15); head black (Figs 8, 12); propleuron rather dark brown; mesoscutum and scutellum black.

..2

2. Scutellum and metanotum without apical spine in lateral view; fore wing veins $r$ and 2-SR curved (Fig. 15); second and third metasomal tergites shiny, smooth (Fig. 14); hind coxa coriaceous dorsally.

Nyereria yenthuyensis Long \& van Achterberg, 2008

- Scutellum and metanotum with apical spine in lateral view, (Fig. 31 in Long \& van Achterberg, 2008) [9]; fore wing veins $\mathrm{r}$ and 2-SR more or less angled (Fig.11); second and third metasomal tergites punctate or rugose (Fig. 10); hind coxa punctate dorsally Nyereria albicentrus Long \& van Achterberg, 2008

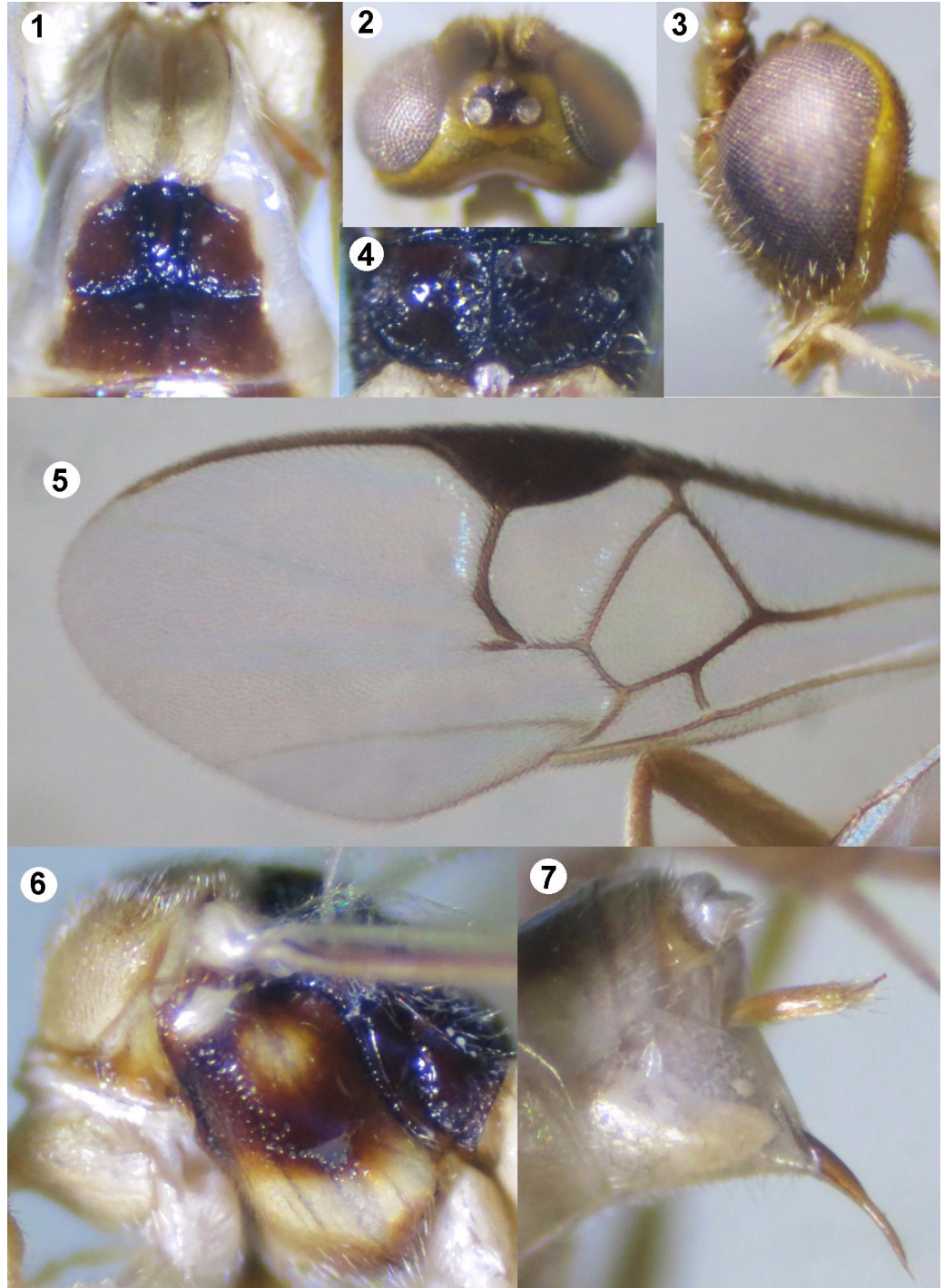

Figures 1-7. Nyereria bicolorata sp. $\mathrm{n}$.

1. First-third metasomal tergites;

2. Head (dorsal view);

3. Head (lateral view);

4. Propodeum;

5. Fore wing;

6. Mesoleuron;

7. Hypopygium. 


\section{Description}

Nyereria bicolorata Long \& van Achterberg, sp. n. (Figs 1-7)

Material: Holotype, $q$ (VNMN), Mic.1119, NE Vietnam: Thai Nguyen, Dai Tu, Cat Ne, MT, garden, $21^{\circ} 31^{\prime} \mathrm{N} 105^{\circ} 39^{\prime} \mathrm{E}, 300 \mathrm{~m}, 20-$ 30.xii.2007, KD Long; paratype, 1 \& (VNMN), Mic.138, VN: Ha Tinh, Huong Son, $18^{\circ} 22^{\prime} \mathrm{N}$ $106^{\circ} 13^{\prime} \mathrm{E}, 450$ m, April 7-13, 1998 AMNH, K. Long.

Description: Body length $3.3 \mathrm{~mm}$, fore wing length $3.0 \mathrm{~mm}$, antenna $4.0 \mathrm{~mm}$.

Head. Third antennal segment as long as fourth; penultimate antennal segment 0.9 times as long as apical segment (7:9); median width of face 0.8 times length of face and clypeus combined (14:18); distance between tentorial pits 4.5 times distance between pits and eyes $(9: 2)$; eyes large, in dorsal view, eye 4.5 times longer than temple (9:2) (fig. 2); in lateral view, width of eye 3.5 times as long as temple (14:4) (fig. 4); ocelli in high triangle, anterior tangent of posterior ocelli not touching anterior ocellus (fig. 2); POL equal to OOL; POL:OD:OOL= 3:3:3; distance between front and hind ocelli 0.5 times OOL. Face punctuate with short median carina bellow antennal sockets; vertex and temple almost smooth with sparse fine punctures.

Mesosoma. Length of mesosoma 1.2 times as long as high (44:38); mesopleuron largely smooth, sparsely punctate anteriorly (fig. 6); precoxal sulcus wide, shallow, sparsely punctate (fig. 6); scutellar sulcus with 6 carinae; mesoscutum largely punctate medio-posteriorly, sparsely punctate laterally; scutellum rugopunctate; propodeum with medial carina, smooth laterally, sparsely punctate near median carina (fig. 3); propodeal spiracle round, small.

Wings. Fore wing: pterostigma 2.5 times as long as wide (25:10); vein $r$ of fore wing shorter parastigma, arising after middle of pterostigma (Fig. 5); vein $\mathrm{r} 1.7$ times as long as vein 2-SR (10:6); 1-CU1 1.3 times as long as vein 2-SR (9:7); r:cu-a:2-CU1=9:5:7; fore vein $2-\mathrm{SR}+\mathrm{M}$ as long as vein $2-\mathrm{SR}$ and 0.8 times vein $\mathrm{m}$-cu (6:7); vein 1-SR+M curved basally (fig. 5).
Legs. Length of hind femur:tibia:basitarsus: tarsus $=38: 41: 24: 50$; length of hind femur, tibia and basitarsus 3.8, 5.8 and 6.0 times as long as their width, respectively; inner hind tibial spur 0.8 times as long as basitarsus (19:24). Hind coxa shiny, punctate dorso-laterally.

Metasoma. First metasomal tergite slightly narrowed apically (fig. 1), 2.0 times as long as apical width; first tergite with median groove, largely smooth with transverse striae at apex; second metasomal tergite with rugose rectangular area (fig. 1); second tergite coriaceous; the rest tergites shiny, smooth. Hypopygium short; ovipositor sheath short with sparse hairs near apex; ovipositor thick basally and abruptly tapered near middle length (fig. 7).

Colour. Palpi ivory; head, antennae, pronotum, mesosternum, mesoscutum and scutellum yellow; tegula yellow; fore and middle legs whitish yellow; hind leg yellow, except hind femur extreme apically and hind tibia basically brownish yellow; wing veins brownish yellow; setae pale yellow; metanotum and propodeum dark brown; first metasomal tergite white; second-sixth tergite brownish yellow.

Variation: A few of colour differences in paratype: antenna brownish yellow; mesoscutum and scutellum pale brown; mesosternum brown; fore and middle legs yellow; hind leg brownish, except hind tibia apically.

\section{Male: Unknown.}

Etymology: From "bi" (Latin for "two") and "coloris" (Latin for "hue, tint"), because of the bi-coloured body.

Host: Unknown.

Distribution: NE Vietnam: Thai Nguyen (Dai Tu); NC Vietnam: Ha Tinh (Huong Son).

Notes: The new species is an aberrant species differing from the other species form Vietnam by having ocelli in a high triangle (fig. 2 ), the eyes large, the temple narrow (in lateral view width of eye 3.5 times temple width: fig. 4 ), the fore wing vein $r$ distinctly shorter than parastigma, vein $1-\mathrm{SR}+\mathrm{M}$ of fore wing curved basally (fig. 5), the propodeum largely smooth 
and only sparsely punctate near median carina (fig. 3), the first metasomal tergite slightly narrowed apically (fig.1) and the head, mesoscutum and scutellum yellow.

Nyereria albicentrus Long \& van Achterberg, 2008 (Figs 8-11) (Figs $1-8$ in Long \& van Achterberg, 2008) [9]

Nyereria albicentrus Long \& van Achterberg, 2008. Tap chi Sinh hoc 2008, 30(3): 82 .
Material: Holotype, +, (IEBR), Apan.740, Vietnam: Ha Tinh (Huong Son), forest, (MT), $18^{\circ} 22^{\prime} \mathrm{N} 106^{\circ} 13^{\prime} \mathrm{E}, 900 \mathrm{~m}$, May 18, 1998, $\mathrm{AMNH}, \mathrm{K}$. Long. Paratypes: 10, (IEBR), Mic.1044, same locality, but forest 900 m, MT, 05.V.1998, AMNH, K. Long; 1, (RMNH), Mic.1023: Vietnam: Ha Tinh (Huong Son), forest, (MT), $18^{\circ} 22^{\prime} \mathrm{N} 106^{\circ} 13^{\prime} \mathrm{E}, 20-28 . I V .1998$, AMNH, K. Long. 1ㅇ, Mic.1621, (VNMN), NE Vietnam: Vinh Phuc, Tam Dao NP, MT, 21 ${ }^{\circ} 27^{\prime} \mathrm{N}$ 105³8'E, 1000 m, 10-20.iv.2012, KD Long.

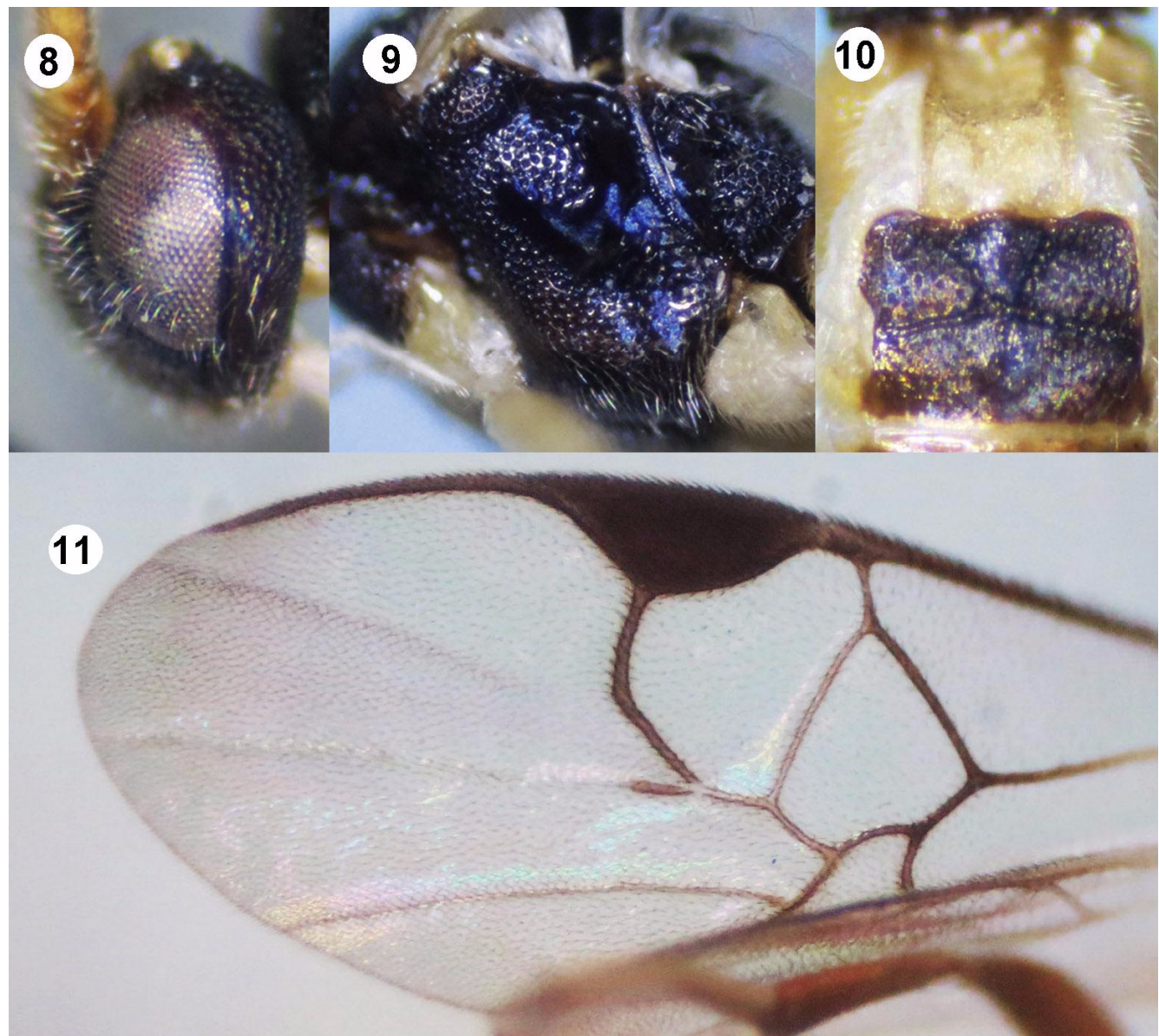

Figures 8-11. Nyereria albicentrus Long \& van Achterberg, 2008

8. Head (lateral view); 9. Mesopleuron; 10. First-third metasomal tergites; 11. Fore wing.

Diagnosis: Ocelli in very low triangle, tangent of posterior ocelli cutting middle anterior ocellus; in dorsal view, eye 1.1 times longer than temple (8:7); in lateral view width of eye 1.6 times as long as temple (fig. 8); vertex and temple rugose punctate; mesopleuron smooth medially, largely punctate anteriorly and ventrally (fig. 9); metapleuron almost punctate; scutellum and mesoscutum with apical spine (fig. 31, Long \& van Achterberg, 2008 [9]); fore wing veins $\mathrm{r}$ and 2-SR weakly angled (fig. 11), vein 1-CU1 subequal to vein 2-CU1; 
second metasomal tergite rectangular, shorter than third tergite (fig. 10); second and third tergites largely punctate; hind coxa pale yellow, rugo-punctate.

Host: Unknown.

Distribution: NE Vietnam: Vinh Phuc (Tam Dao NP); NC Vietnam: Ha Tinh (Huong Son).

Nyereria yenthuyensis Long \& van Achterberg, 2008 (Figs 12-15); (Figs 9- 16 in Long \& van Achterberg, 2008) [9]

Nyereria yenthuyensis Long \& van Achterberg, 2008. Tap chi Sinh hoc 2008, 30(3): 83 .
Material: Holotype, + , (IEBR), Mic.918, Vietnam: Hoa Binh (Yen Thuy), (MT), forest, $20^{\circ} 28^{\prime} \mathrm{N} 105^{\circ} 34^{\prime} \mathrm{E}, 20-30 . X .2003$, KD Long.

Diagnosis: Tangent of posterior ocelli touching anterior ocellus; in dorsal view, eye 1.4 times longer than temple $(7: 5)$; in lateral view width of eye 1.7 times as long as temple (fig. 12); vertex and temple sparsely punctate; mesopleuron largely smooth medially, punctate anteriorly (fig. 13); metapleuron largely smooth; scutellum and mesoscutum without apical spine; fore wing veins $r$ and 2-SR curved (fig. 15); second metasomal tergite as long as third tergite; second and third tergites, shiny, smooth (fig. 14); hind coxa black, coriaceous.

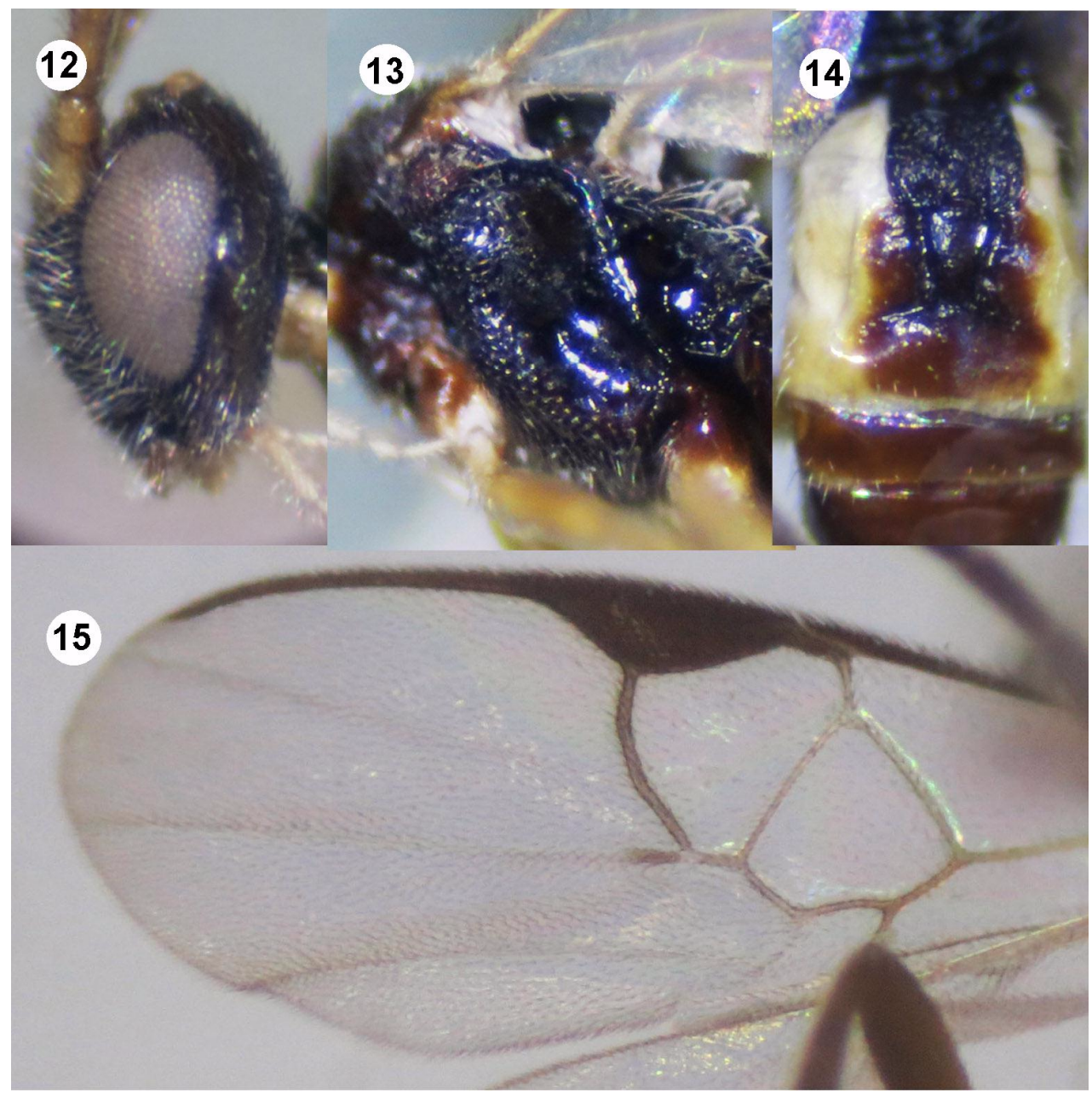

Figures 12-15. Nyereria yenthuyensis Long \& van Achterberg, 2008

12. Head (lateral view); 13. Mesopleuron; 14. First-third metasomal tergites; 15. Fore wing. 


\section{Host: Unknown.} Thuy).

Distribution: NW Vietnam: Hoa Binh (Yen

Acknowledgements: This paper has been produced with the fund of the Vietnam National Foundation for Science and Technology Development (NAFOSTED, grant No. 106.152012.62).

\section{REFERENCES}

1. Achterberg van C., 1988. Revision of the subfamily Blacinae Foerster (Hymenoptera, Braconidae). Zoologische Verhandelingen Leiden, 249: 1-324.

2. Austin A. D., Dangerfield P. C., 1992. Synopsis of Australasian Microgastrinae (Hymenoptera: Braconidae), with a key to genera and description of new taxa. Invertebrate Taxonomy, 6(1): 1-76.

3. De Saeger H., 1944. Microgasterinae (Hymenoptera: Apocrita). Exploration du Parc National Albert. Mission G.F. de Witte, 47: 1-342.

4. Granger C., 1949. Braconides de Madagascar. Memoires de l'Institut Scientifique de Madagascar, (A)2:1-428.

5. Harris R. A., 1979. A glossary of surface sculpturing. Occasional Papers in Entomology, California Department of Food and Agriculture, 28: 1-33.

6. Khuất Đăng Long, 2007. Hai loài mới cho khoa học thuộc giống Ong kén trắng Apanteles Foerster, 1862 (Hymenoptera: Braconidae: Microgastrinae) ở Việt Nam, Tạp chí Sinh học, 29(3): 25-31.

7. Khuat Dang Long, 2010. Five new species of the genus Apanteles (Hymenoptera: Braconidae: Microgastrinae) from Vietnam. Tap chi Sinh học, 32(4): 69-79.

8. Khuat Dang Long, Belokobylskij S. A., 2003. A preliminary list of the Braconidae
(Hymenoptera) of Vietnam, Russian Entomological Journal, 12(4): 385-398.

9. Khuat Dang Long, Cornelis van Achterberg, 2008. Two genera and one species newly recorded with description of five species of the subfamily Microgastrinae (Hymenoptera: Braconidae) from Vietnam. Tap chi Sinh hoc, 30(3): 78-87.

10. Khuat Dang Long, Achterberg C. van, 2014. An additional list with new records of braconid wasps of the family braconidae (Hymenoptera) from Vietnam. Tap chi Sinh hoc, 36(4): 397-415. DOI: 10.15625/08667160/v36n4.5979.

11. Mason W. R. M., 1981. The polyphyletic nature of Apanteles Foerster (Hymenoptera: Braconidae): A phylogeny and reclassification of Microgastrinae. Mem. Entom. Soc. Canada, 115: 1-147.

12. Nixon G. E. J., 1965. A reclassification of the tribe Microgasterini (Hymenoptera: Braconidae). Bull. Br. Mus. Nat. Hist. Entomology, Suppl. 2: 1-284.

13. Rousse P., Gupta A., 2013. Microgastrinae (Hymenoptera: Braconidae) of Reunion Island: a catalogue of the local species, including 18 new taxa and a key to species. Zootaxa, 3616(6): 501-547. DOI: 10.11646/zootaxa.3616.6.1.

14. Sathe T. V., Inamdar S. A., 1988. A new species of the genus Nyereria Wilkinson (Hymenoptera: Braconidae) from India. Journal of Advanced Zoology, 9(2): 128-131.

15. Tobias V. I., 1977. New species of braconids of the genus Apanteles Forst. (Hymenoptera, Braconidae) from the Far East. Trudy Zoologicheskogo Instituta. Leningrad, 67(1976): 90-96 (in Russian).

16. Yu D. S. K., Achterberg C. van, Horstmann K., 2012. Biological and taxonomical information: Ichneumonoidea 2012. Taxapad Interactive Catalogue, Ottawa. Database on flash-drive. www.taxapad.com. 


\title{
MỘT LOÀI MỚI CHO KHOA HỌC THUỘC GIỐNG Nyereria Mason (Hymenoptera: Braconidae: Microgastrinae) TỪ KHU HỆ CỦA VIẸT NAM
}

\author{
Khuất Đăng Long ${ }^{1}$, Cornelis Van Achterberg ${ }^{1}$ \\ ${ }^{1}$ Viện Sinh thái và Tài nguyên sinh vật, Viện Hàn lâm KH \& CN Việt Nam \\ ${ }^{2}$ Department of Terrestrial Zoology (RMNH), Naturalis Biodiversity Center, Leiden, Netherlands
}

\section{TÓM TẮT}

Giống Nyereria Mason, 1981 thuộc phân họ ong ký sinh Microgastrinae, họ Braconidae có số loài không nhiều và được biết đến chủ yếu từ vùng nhiệt đới châu Phi, khu hệ của vùng này đã biết 19 loài. Ở Việt Nam, đến nay mới biết 2 loài, đó là Nyereria albicentrus Long \& van Achterberg, 2008 and N. yenthuyensis Long \& van Achterberg. Hai loài này trước đây được mô tả dưới tên phân giống Nyereria Mason thuộc giống Protapanteles Ashmead. Trong bài báo này, một loài mới cho khoa học, Nyereria biolorata Long \& van Achterberg, sp. n., được mô tả và minh họa từ mẫu vật được thu thập ở Thái Nguyên (Đại Từ) và Hà Tĩnh (Hương Sơn). Những khác biệt về đặc điểm hình thái của loài mới đã được chỉ ra trong khóa định loại và chẩn loại cho 3 loài thuộc giống Nyereria ở Việt Nam.

Cho đến nay, chưa có thông tin về vật chủ của 3 loài ong ký sinh này ở Việt Nam, tuy nhiên, dựa theo những thông tin về vật chủ của các loài ong ký sinh thuộc phân họ Microgastrinae chủ yếu là sâu non các loài thuộc họ cánh Vảy (Lepidoptera), điều này cũng không thuộc ngoại lệ đối với các loài thuộc giống Nyereria. Mẫu chuẩn (holotype và paratype) được lưu giữ tại Bảo tàng Thiên nhiên Việt Nam (VNMN).

Tù khóa: Nyereria, bộ Cánh màng, loài mới, ong ký sinh.

Ngày nhận bài: 9-5-2015 